\title{
Association of Tortuous Common Carotid Artery with Abnormal Distribution of the Ansa Cervicalis: A Case Report
}

\author{
George Paraskevas ${ }^{1, *}$, Konstantinos Koutsouflianiotis', Chrysanthos Chrysanthou ${ }^{1}$, Kalliopi Iliou ${ }^{1}$, \\ Nikolaos Syrmos ${ }^{1}$, Marios Salmas ${ }^{2}$
}

ABSTRACT

In the current study, we display a rare association of an aberrant innervation of the sternocleidomastoid muscle by the ansa cervicalis (AC) with a tortuous common carotid artery (TCCA). In specific, in a male cadaver we observed on the right side of the cervical region, a nerval branch of remarkable size originating from the most distal part of the AC's superior root and after piercing the superior belly of the omohyoid muscle innervated the distal portion of the sternocleidomastoid muscle. Furthermore, we noticed a tortuous course of the initial part of the right common carotid artery. We discuss the surgical significance of the awareness of AC's variations during neurotisation of the recurrent laryngeal nerve in cases of its damage, as well as the importance of aberrant innervation of the sternocleidomastoid muscle by AC for the preservation of muscle's functionality after accessory nerve's damage. Furthermore, we highlight the fact, that the knowledge of the relatively uncommon variant, such as TCCA is crucial for the physician in order to proceed more effectively in differential diagnosis of a palpable mass of the anterior cervical region or deal with symptoms such as dyspnea, dysphagia or symptoms of cerebrovascular insufficiency.

\section{KEYWORDS}

ansa cervicalis; common carotid artery; tortuosity; sternocleidomastoid muscle; variations

AUTHOR AFFILIATIONS

${ }^{1}$ Department of Anatomy and Surgical Anatomy, School of Medicine, Aristotle University of Thessaloniki, Thessaloniki, Greece

2 Department of Anatomy and Surgical Anatomy, School of Medicine, National and Kapodistrian University of Athens, Athens, Greece

* Corresponding author: Department of Anatomy and Surgical Anatomy, School of Medicine, Aristotle University of Thessaloniki, Thessaloniki, Greece; g_paraskevas@yahoo.gr

Received: 13 July 2020

Accepted: 29 January 2021

Published online: 30 July 2021

Acta Medica (Hradec Králové) 2021; 64(2): 129-131

https://doi.org/10.14712/18059694.2021.22

(c) 2021 The Authors. This is an open-access article distributed under the terms of the Creative Commons Attribution License (http://creativecommons.org/licenses/by/4.0), which permits unrestricted use, distribution, and reproduction in any medium, provided the original author and source are credited. 


\section{INTRODUCTION}

The ansa cervicalis (AC) or ansa hypoglossi as it is known is a cervical loop usually formed by first to third cervical nerves and innervating the infrahyoid muscles (1). There is a variability in regards to the cervical nerves which participate in its formation, the length of the loop with respect to the omohyoid muscle, the variable morphology of the AC's inferior root, the AC's relationship to the internal jugular vein or the likely participation of the vagus nerve in $\mathrm{AC}$ formation (2). However, the presence of additional AC's branches distributed to the adjacent muscles, such as the sternocleidomastoid muscle is rarely detected in the literature (3-4). As regards the tortuous common carotid artery (TCCA) is an usually asymptomatic elicosis of the common carotid artery of unclear origin which rarely may be palpated as a mass of the anterior cervical region or very rarely may be associated with symptoms of cerebrovascular insufficiency (5-6).

In the current study, we display a rare combination of an aberrant innervation of the sternocleidomastoid muscle by the AC, along with a TCCA, which is an interesting opportunity to search through the literature for similar anatomical variations.

\section{CASE REPORT}

During the routine dissection of the neck in our Department of Anatomy in a 82-year-old male cadaver whose cause of death was unrelated to the current case study, an infrequent combination of an aberrant innervation of the sternocleidomastoid muscle by the AC along with TCCA was detected. In particular, after meticulous dissection of the anterior, as well, lateral cervical region we observed on the right side, a nerval branch of remarkable size originating from the most distal part of the AC's superior root and

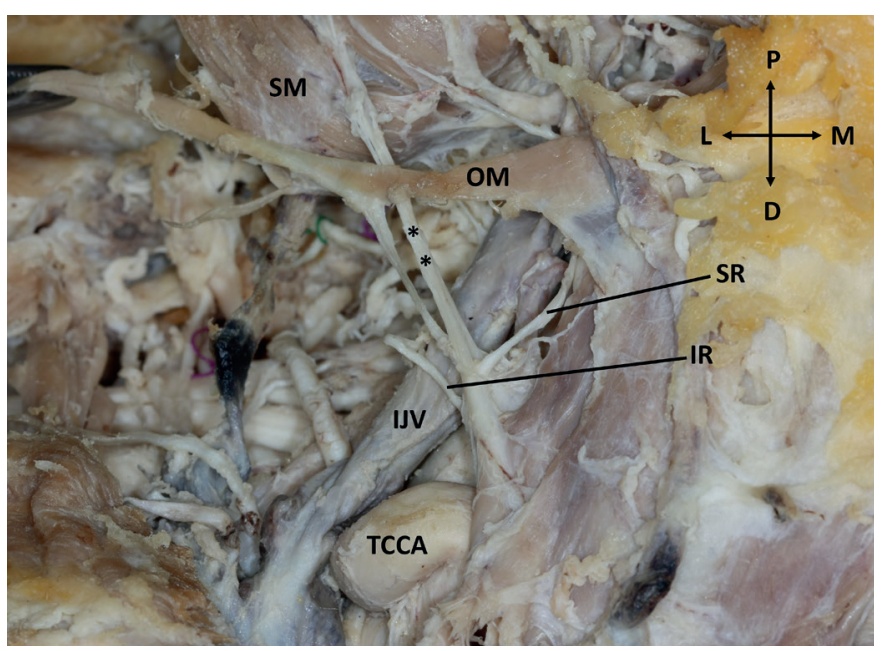

Fig. 1 On the right hemi-neck an aberrant branch $\left({ }^{* *}\right)$ of the ansa cervicalis' superior root (SR) is seen piercing the superior belly of the omo-hyoid muscle (OM) and distributing to the inferior segment of the sterno-cleido-mastoid muscle (SM). The initial portion of the right common carotid artery displays a tortuous course (TCCA) (IR: inferior root of ansa cervicalis, IJV: internal jugular vein, P: proximal, D: distal, L: lateral, M: medial). after piercing the superior belly of the omohyoid muscle innervated the distal portion of the sternocleidomastoid muscle. Furthermore, we noticed a tortuous course of the initial part of the right common carotid artery (Figure 1). No other anatomical variations were present, or evidence of previous surgical procedures undertaken on the neck. The detected nerval and vascular variations, along with their relationship to the adjacent anatomical elements were documented by photographs.

\section{DISCUSSION}

The AC as it is widely known is a neural loop formed by the union of a superior root originating from the ventral ramus of first cervical nerve (joining the hypoglossal nerve) and the inferior root being formed by the union of the ventral rami of the second and third cervical nerve. Ultimately, that nerval loop provides innervation to the infrahyoid muscles (1). There is a plethora of AC's variation concerning the pattern of cervical roots participating in its formation, the AC's length with respect to omohyoid muscle (proximal or distal to it), the various forms of AC in regard to the inferior root, the AC's relationship to the internal jugular vein, or the possible neural communications between cervical component ( $\mathrm{C} 1-\mathrm{C} 2$ fibers), vagal component ( $\mathrm{C} 1-\mathrm{C} 2$ fibers with the vagus nerve) and hypoglossal component (C1-C2 fibers with the hypoglossal nerve) (2). However, we consider that the presentation of classification of such morphological AC's patterns is beyond the aim of the current study.

Apart from the AC's formation from cervical fibers derived from the hypoglossal and vagus nerves, there are cases in which the cervical fibers of the AC are derived from the accessory nerve (7). Furthermore, a case of AC's formation by a cervical branch to the sternocleidomastoid muscle has been previously prescribed (8). It should be emphasized that the AC might be differentiated from the so-called "pseudo-ansa cervicalis", in which the superior root arises from the vagus nerve, whereas the inferior root from the superior cervical sympathetic ganglion (9).

Beyond the classic AC's branches to the infrahyoid muscles, there are reports in which the AC provides rami to the cardiac or phrenic nerves (3). The case in which the AC provides branch or branches to the adjacent sternocleidomastoid muscle, as occurs in the present study, has been very rarely detected in the literature. A first mention of such a branch has been brought by Piersol in 1911 (10). Later, Koizumi et al noticed a case in which the AC's superior root, provided a branch innervating the sternocleidomastoid muscle (3). Paraskevas et al presented a case of a nerve innervating the sternocleidomastoid muscle; that nerve was formed after the union of two rami of the AC's branch to the sternothyroid muscle (4). Blythe at al observed a nerve, similar in diameter to the accessory nerve that innervated the lower third of the sternocleidomastoid muscle (11). That nerve arised from the hypoglossal nerve proximal to the origin of the hypoglossal branch. In the current study a nerve trunk of remarkable size was seen arising from the most distal portion of the AC's superior root and after piercing the superior belly of the omohyoid muscle was distributed to the distal segment of the sternocleidomastoid muscle. 
Due to close location of AC to recurrent laryngeal nerve, the AC may be utilized for neurotisation of that nerve, since the recurrent laryngeal nerve is active during phonation. The recurrent laryngeal nerve may be damaged during various surgical procedures undertaken in the region, such as oesophageal cancer surgery (12). Furthermore, AC can be used also in preventing the morbidity associated with tongue hemiatrophy after facial-hypoglossal anastomosis $(13,14)$ or as a donor, during neurotisation of brachial plexus (descending and ascending loop of AC) (15). Although AC's sacrifications does not induce serious functional consequences, it should be noted that the infrahyoid muscles which are innervated by the AC, play a role in laryngeal steadiness during phonation and deglutition (16).

The TCCA is an anatomical variant of unclear origin, usually accidentally detected during a surgical procedure or imaging of the neck or rarely observed as a mass of the cervical region. As regards the various types of spiral course of carotid arteries, three types of tortuosity have been described by Weibel et al, the tortuosity type, as an $\mathrm{s}$ - or c-shaped elongation, the kinking type as an acute angulation and the looping type as an exaggerated s-shaped curve resembling to a circular course (17). The TCCA is usually asymptomatic and accidentally detected in imaging of the region. In some instances individuals may feel a pulsating mass in the anterior cervical region (18). Rarely, patients should have a painful sensation, progressive dysphagia or dyspnea or symptoms of cerebrovascular insufficiency (5), or TCCA may be masquerading as a thyroid mass (6) or as a submandibular mass (19).

It must be emphasized that TCCA usually does not result in cerebrovascular disease; most commonly a TCCA aneurysm, usually of traumatic origin can lead to such pathologic condition. Usually, TCCAs do not display atherosclerotic lesions, however, the hypertension and the atherosclerotic lesions result in aortic arch elevation and a subsequent formation of a common carotid artery looping $(5,18)$. Predisposing factors associated with TCCA are old age, female sex, hypertension and other cardiovascular risk factors, whereas associated diseases usually genetic syndromes are among others, Marfan syndrome, arterial tortuosity syndrome, fibromuscular dysplasia and other inherited arteriopathies (20).

The diagnosis of a TCCA requires physical examination and imaging, such as ultrasonography with Doppler color flow image, computed tomography, magnetic resonance imaging, angiography and especially computed tomographic angiography (6). As regards the treatment options of TCCA, it is generally accepted that there is no need for a specific therapy. It has been shown that treatment of the likely associated hypertension could potentially reduce the dimensions of the TCCA's mass. Uncommonly, and especially in cases of existence of symptoms of cerebrovascular disease, a graft should be inserted after TCCA resection (5).

\section{CONCLUSION}

The precise awareness of AC's morphology and topography is crucial for the surgeon of the region in order not to damage it, since AC is utilized as a graft for larynx's re-innervation following recurrent laryngeal nerve paralysis. Moreover, the knowledge of partial innervation of the sternocleidomastoid muscle from the AC is essential for the surgeon of the region, since it can explain almost the total functionality of that muscle in potential laceration of the accessory nerve during lymph nodes' dissection at the cervical region. Furthermore, the possible presence of TCCA should be kept in mind of physician dealing with palpable masses of the anterior cervical region or dealing with differential diagnosis of dysphagia or dyspnea. Neurologists and neurosurgeons should be also aware of such anatomic variant, as a possible cause of cerebrovascular insufficiency or during carotid endarterectomies.

\section{REFERENCES}

1. Williams PL. Gray's anatomy. The anatomical basis of medicine and surgery. 38th ed. London: Churchill Livingstone, 1995: 1257-8.

2. Kikuta S, Jenkins S, Kusukawa J, Iwanaga J, Loukas M, Tubbs RS. Ansa cervicalis: a comprehensive review of its anatomy, variations, pathology, and surgical applications. Anat Cell Biol 2019; 52: 221-5.

3. Koizumi M, Horiguchi M, Sekiya S, Isogai S, Nakano M. A case of the human sternocleidomastoid muscle additionally innervated by the hypoglossal nerve. Okajimas Folia Anat Jpn 1993; 69(6): 361-7.

4. Paraskevas G, Natsis K, Nitsa Z, Mavrodi A, Kitsoulis P. Unusual morphological pattern and distribution of the ansa cervicalis: a case report. Rom J Morphol Embryol 2014; 55(3): 993-6.

5. Leipzig TJ, Dohrmann GJ. The tortuous or kinked carotid artery: pathogenesis and clinical considerations. Surg Neurol 1986; 25: 478-86.

6. Chen PJ, Chen HC. Tortuous common carotid artery masquerading as thyroid mass. Clin Surg 2018; 3: 2070.

7. Quadros LS, Bhat N, Babu A, D'souza AS. Anatomical variations in the ansa cervicalis and innervations of infrahyoid muscles. Int J Anat Res 2013; 1(2): 69-74.

8. Khaki AA, Shokouhi G, Shoja MM, et al. Ansa cervicalis as a variant of spinal accessory nerve plexus: a case report. Clin Anat 2006; 19: 540-3.

9. Indrasingh I, Vettivel S. A rare pseudo ansa cervicalis: a case report. J Anat Soc India 2000; 49: 178-9.

10. Piersol GA. Human anatomy. 3d edn. Philadelphia: JB Lippincott, 1911.

11. Blythe JNStJ, Matharu J, Reuther WJ, Brennan PA. Innervation of the lower third of the sternocleidomastoid muscle by the ansa cervicalis through the $\mathrm{C} 1$ descendens hypoglossal branch: a previously unreported anatomical variant. Br J Oral Maxillofac Surg 2015; 53: 470-1.

12. Loukas M, Thorsell A, Tubbs RS, et al. Folia Morphol 2007; 66(2): 120-5.

13. Kukwa A, Marchel A, Pietniczka M, Rakowicz M, Krajewski R. Reanimation of the face after facial nerve palsy resulting from resection of a cerebellopontine angle tumour. Br J Neurosurg 1994; 8(3): 327-32.

14. Laurentjoye M, Ricard AS, Caix P, Siberchicot F, Majoufre-Lefebvre C. Le lambeau infrahyoïdien bilatéral innervé par l'Ansa Cervicalis pour la reconstruction des glossectomies totales [Tongue reconstruction with a bilateral infrahyoid flap innervated by Ansa Cervicalis after total glossectomy]. Rev Stomatol Chir Maxillofac 2011; 112(6): 337-41.

15. Amr SM, Moharram AN, Abdel-Meguid KM. Augmentation of partially regenerated nerves by end-to-side side-to-side grafting neurotization: experience based on eight late obstetric brachial plexus cases. J Brachial Plex Peripher Nerve Inj 2006; 1: 6.

16. Chhetri DK, Berke GS. Ansa cervicalis nerve: review of the topographic anatomy and morphology. Laryngoscope 1997; 107: 1366-72.

17. Weibel J, Fields WS. Tortuosity, coiling and kinking of the internal carotid artery. I. Etiology and radiographic anatomy. Neurology 1965; 15: 7-18.

18. Iwanaga J, Watanabe K, Tsuyoshi S, Tabira Y, Yamaki K. Tortuous common carotid artery: a report of four cases observed in cadaveric dissections. Case Rep Otolaryngol 2016; 2016: 2028402.

19. Xu C, Uwiera TC. Tortuous common carotid artery presenting as a pediatric submandibular neck mass. Int J Pediatr Otorhinolaryngol Extra 2010; 5: 53-6.

20. Ciurica S, Lopez-Sublet M, Loeys BL, et al. Arterial tortuosity. Novel implications for an old phenotype. Hypertension 2019; 73: 951-60. 